\title{
Mitochondrial network in the heart
}

\author{
Qian Li, Lu-Yu Zhou, Gui-Feng Gao, Jian-Qin Jiao, Pei-Feng Li ${ }^{凶}$ \\ Division of Cardiovascular research, State Key Laboratory of Biomembrane and Membrane Biotechnology, Institute of Zoology, \\ Chinese Academy of Sciences, Beijing 100101, China \\ $\bowtie$ Correspondence: peifli@ioz.ac.cn \\ Received March 14, 2012 Accepted March 17, 2012
}

\begin{abstract}
Mitochondria are subcellular organelles that provide energy for the cell. They form a dynamic tubular network and play an important role in maintaining the cell function and integrity. Heart is a powerful organ that supplies the motivation for circulation, thereby requiring large amounts of energy. Thus, the healthiness of cardiomyocytes and mitochondria is necessary for the normal cardiac function. Mitochondria not only lie in the center of the cell apoptotic pathway, but also are the major source of reactive oxygen species (ROS) generation. Mitochondrial morphological change includes fission and fusion that are regulated by a large number of proteins. In this review we discuss the regulators of mitochondrial fission/fusion and their association with cell apoptosis, autophagy and ROS production in the heart.
\end{abstract}

KEYWORDS mitochondrial network, heart diseases, reactive oxygen species

\section{INTRODUCTION}

It is known that heart diseases have become a leading cause of mortality in our society, especially in developed countries. The heart diseases include coronary heart disease, cardiomyopathy, chemotherapeutic drug-induced cardiotoxicities, myocarditis, the cardiac complications of muscular dystrophy and diabetes (Baines, 2010). The large increase of morbidity and mortality due to cardiac diseases makes their preventions and therapies an urgent issue to be investigated.

Heart is the most dynamic organ, and it requires large amounts of energy. It is of note that the mitochondria take up as much as $30 \%$ of total cardiomyocyte volume. Due to the limited ability for substrate storage, the heart function strin- gently depends on the ATP-generating pathway (Huss and Kelly, 2005). Mitochondria provide the energy required for cardiomyocytes function (Finck and Kelly, 2007). Mitochondrial number in the cardiomyocytes is dynamically regulated in response to cardiac energy demands (Lehman et al., 2000). For example, the number of mitochondria in the cardiomyocytes substantially increases due to the great energy demands (Mayor and Cuezva, 1985). Thus, the high abundant existence of functional mitochondria in cardiomyocytes is essential in producing enough ATP required for normal cardiac contractile function (Lin et al., 2001). In addition, mitochondria participate in the maintaining of ion and reactive oxygen species (ROS) homeostasis. On the contrary, there are abundant studies showing that the injured and abnormal mitochondria can bring harmful effects on the cardiomyocytes. It has been identified that mitochondria are greatly involved in the onset and deterioration of diverse cardiac diseases.

In this review, we summarize the relevance of mitochondrial network in heart in aspects of mitochondrial morphology, apoptosis and ROS. In particular, we discuss the relationship between mitochondrial malfunction and cardiac diseases.

\section{MITOCHONDRIAL MORPHOLOGY IN HEART}

Cardiomyocytes are enriched in mitochondria, which form an interconnected network within the cells. Those mitochondria in contact with the sarcolemma are subsarcolemmal mitochondria (SSM) with lamelliform cristae, while interfibrillar mitochondria (IFM) situated deeper in the cells without obvious contact with the plasma membrane (Hoppela et al., 2009). Mitochondria are highly dynamic organelles that constantly undergo fusion and fission. The abnormal fusion and fission can lead to the cardiac disorders (Li et al., 2010; Wang et al., $2011 \mathrm{~b})$. The maintenance of mitochondrial morphological integrity is vitally important for the cardiac structure and function. 


\section{MITOCHONDRIA FISSION AND FUSION}

Mitochondrial fusion and fission are opposing processes that control mitochondrial number, morphology and, to some extent, mitochondrial quality (Twig et al., 2008b) (Fig. 1). Through fission, mitochondria divide into daughter organelles, resulting in an elevation of the mitochondrial number (Detmer and Chan, 2007). Mitochondrial fusion mixes mitochondria contents, thus enabling protein complementation (Detmer and Chan, 2007). The disruption of fusion leads to the fragmentation of the mitochondrial tubular network (Griparic et al., 2004), while abnormal fission causes elongated and interconnected tubules (Stojanovski et al., 2004).

As a selective process, mitochondria fusion allows healthy mitochondria to fuse with each other (Hyde et al., 2010). The regulators of mitochondrial fusion in human include a variety of proteins such as mitofusin-1 (Mfn-1), mitofusin-2 (Mfn-2) and the optic atrophy-1 (OPA1) protein (Detmer and Chan, 2007; Chen and Chan, 2004). These proteins are dynamin-related GTPases. Mfn-1 and Mfn-2 are located in the mitochondrial outer membrane, and interact with each other to direct the fusion of the neighboring mitochondrial outer membrane (Eura et al, 2003). OPA1 resides in the mitochondrial intermembrane space and mediates the inner membrane fusing (Cipolat et al., 2004).

Unlike fusion, fission is a nonselective process (Twig et al., 2008b). Dynamin-related protein 1 (Drp1) and Fis1 are required for mitochondrial fission in mammals. In healthy mammalian cells, endogenous Drp1 is predominantly localized in the cytosol and can be translocated into mitochondria during apoptosis. Overexpression of dominant negative Drp1 protein results in dramatic increase of mitochondrial connectivity (Smirnova et al., 2001), while overexpression of Drp1 promotes mitochondrial fission and increases cell vulnerability (Cribbs and Strack, 2007). In contrast to Drp1, Fis1 is exclusively localized to mitochondria and overexpression of Fis1 in HeLa cells can promote extensive mitochondrial fragmentation (Jofuku et al., 2005). The role of Fis1 in mitochondrial fission program in mammalian cells remains to be further uncovered.

\section{MITOCHONDRIA AND HEART DISEASES}

The heart is an organ with a high demand of energy, and relies heavily on mitochondria to generate ATP through the oxidative phosphorylation chain. In a variety of cardiac diseases, mitochondria exhibit the abnormal morphology with a small size and fragmentation because of excess fission (Schaper et al., 1991; Hom and Sheu, 2009). A recent research demonstrates that the pharmacological inhibition of the mitochondrial fission protein Drp1 can protect adult murine cardiomyocytes against ischemia/reperfusion injury and reduce myocardial infarct size in the murine heart (Ong et al., 2010). Another research reveals that mitochondria in the neonatal and adult cardiomyocytes undergo rapid and transient fragmentation in response to thapsigargin- or

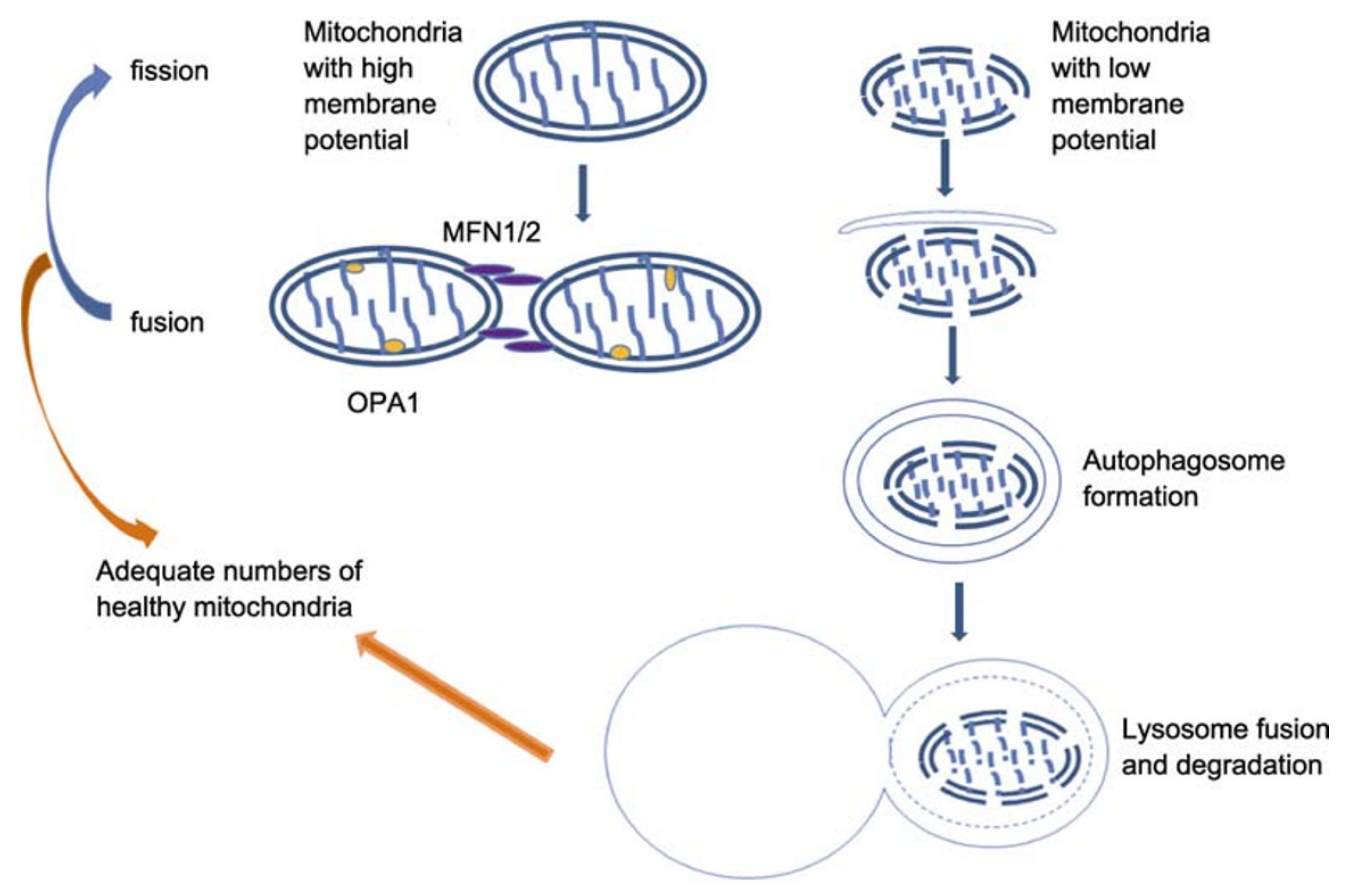

Figure 1. Mitochondrial fission, fusion and mitophagy/autophagy. A mitochondrion is divided into daughter mitochondria, some with high membrane potential and easily to fuse with other healthy mitochondria, others with low membrane potential and easily to be encompassed by autophagosome and then be degraded after autophagsome fuses with lysosome. 
$\mathrm{KCl}$-induced cytosolic calcium increase (Hom et al., 2010). As calcium overload is a character of heart failure, this calcium increment can enhance mitochondria fission and aggravate heart injury. All these findings suggest that manipulating mitochondrial morphology may provide a novel therapeutic strategy for cardiac protection.

\section{MITOCHONDRIA MORPHOLGY MEDIATE MITOPHAGY IN HEART}

\section{Mitophagy in the heart}

During mitochondrial fission, a single mitochondrion divides into daughter units. The larger ones have a high membrane potential and a high probability for subsequent fusion, whereas the smaller ones have a low respiration activity and a low membrane potential (Twig et al., 2008a). Mitochondrial fission requires an intact membrane potential (Legros et al., 2002; Meeusen et al., 2004), the smaller and depolarized mitochondria cannot fuse with the other mitochondria (Twig et al., 2008a; Mouli et al., 2009), thereby leaving the cycle and being degraded by a process termed autophagy (Twig et al., 2008a). Autophagy is a process of catabolism of cellular components including protein aggregates, organelles and viruses. It is necessary for the maintenance of cellular homeostasis (Mizushima et al., 2008; Mortensen et al., 2010; Yang and Klionsky, 2010). The morphological character of autophagy is autophagsome formation that encompasses the cellular components, and fuses with lysosome to degrade it.

Mitophagy selectively degrades mitochondria (Kim et al., 2007). It regulates mitochondria number to match the metabolic or developmental demands. In addition, it removes the damaged mitochondria (Tolkovsky et al., 2002; Kundu et al., 2008). Pink1 and Parkin form an axis that regulates mitophagy in mammalian cells. Pink1 triggers Parkin translocation from cytosol to mitochondria where Parkin ubiquitinates mitochondrial proteins to form autophagosomes (Geisler et al., 2010). In red blood cells, for example, Nix may recruit isolated membranes to mitochondria (Schweers et al., 2007), a process in which UNC51-like kinase1 (ULK1) and Atg7 are involved in the removal of mitochondria (Kundu et al., 2008; Mortensen et al., 2010).

The physiological and pathological significance of mitophagy/autophagy in the heart remains to be elucidated. On one hand, it can remove the impaired mitochondria produced in the pathogenesis of cardiac diseases and thus, is beneficial for cardiac function. For example, the cardiac specific deficiency of Atg5 can lead to cardiac hypertrophy with mitochondrial misalignment and aggregation (Nakai et al., 2007). However, excessive mitophagy/autophagy leads to cardiac damage. Short-term nutrient deprivation induces cardiomyocyte autophagy, and heart failure is accompanied by an increase in autophagy. Disruption of Beclin-1 in mice reduces cardiomyocyte autophagy and abolishes the pathological remodeling in response to the pressure stress (Zhu et al., 2007).

The current understanding appears to be that the mild autophagy in physiological conditions is protective to the heart, while excessive autophagy under the pathological condition is detrimental to the heart (Nakai et al., 2007; Matsui et al., 2007; Zhu et al., 2007).

\section{Mitochondrial morphological changes in mitophagy/autophagy}

The process of mitophagy involves several mitochondrial fission and fusion regulators. Overexpression of Fis 1 leads to extensive fission of mitochondria and induces excessive mitochondrial autophagy (Gomes and Scorrano, 2008). Mitochondrial fusion protein OPA1 deficiency is associated with increased autophagy (White et al., 2009). Under starvation conditions the mitochondrial outer membrane serves as a resource of autophagosome and Mfn-2 plays an important role in regulating the starvation-induced autophagy (Hailey et al., 2010).

In adult cardiac myocytes, Drp1-mediated mitochondrial fission is required for mitophagy induced by Bnip3. Overexpression of dominant negative of Drp1 results in decreased mitochondrial fission and decreased mitophagy (Lee et al., 2011). The relationship between mitochondrial morphology and mitophagy/autophagy in heart is needed to be further investigated.

\section{MITOCHONDRIA MEDIATE APOPTOSIS IN HEART}

Cardiomyocytes are terminally differentiated cells with limited proliferative ability. Their proliferation mechanisms have been explored recently. The loss of cardiomyocytes through apoptosis remains a predominant cause in a variety of cardiovascular diseases. For example, apoptosis has been detected in the animal model of ischemia/reperfusion (Gao et al., 2008), as well as in the patients with acute myocardial infarction (Olivetti et al., 1996), dilated cardiomyopathy (Aharinejad et al., 2008), and metabolic cardiomyopathy (Gürtl et al., 2009). The transgenic mice with tissue specific expression of tumor necrosis factor- $\alpha$ in cardiomyocytes can induce severe pathogenesis of cardiac diseases (Bryant et al., 1998). Thus, apoptosis plays an important role for the pathogenesis of cardiac diseases.

Apoptosis can be mediated by the extrinsic and intrinsic pathways, and mitochondria lie in the core of intrinsic apoptosis pathway. Upon ischemia/reperfusion or oxidative stress injury, the proapoptotic proteins, such as Bax and Bak, translocate to the mitochondrial outer membrane where they impair the permeability of the outer membrane, and induce the release of proapoptotic factors including cytochrome $\mathrm{c}$, Smac/DIABLO or Omi/HtrA2 into the cytoplasm. Cytochrome $\mathrm{c}$ binds to the cytosolic protein Apaf-1 and activates the sub- 
sequent caspases. Smac/DIABLO or Omi/HtrA2 can activate caspases through binding to apoptosis inhibitors (Kroemer et al., 2007; Gustafsson and Gottlieb, 2008). Other Bcl-2 family members such as Bcl-2, Bcl-XL, Bcl-w, Bid, Bim and Puma are also involved in this process (Adams and Cory, 2001). Mitochondrial protein Nix triggers apoptotic cardiomyopathy (Yussman et al., 2002), and Nix overexpression induces cardiomyocyte apoptosis (Diwan et al., 2008).

Preventing cardiomyocyte apoptosis is an important therapeutic target in treating heart diseases. Bifunctional apoptosis regulator (BAR) is an endoplasmic reticulum protein that inhibits cardiomyocyte apoptosis through interacting with the intrinsic apoptosis pathway (Chua et al., 2009). Bcl-2 protects cardiomyocyte from apoptosis (Misao et al., 1996; Imahashi et al., 2004). And cardiac specific Bcl-2 transgenic mice exhibit reduced infarct size and cardiomyocyte apoptosis (Brocheriou et al., 2000; Chen et al., 2001). Apoptosis repressor with caspase recruitment domain (ARC) translocates to mitochondria upon phosphorylation by protein kinase CK2 (Li et al., 2002). It protects the heart from ischemia/reperfusion injury by inhibiting Bax activation (Donath et al., 2006). Taken together, mitochondria play an important role in controlling apoptosis in the heart.

Currently, the relationship between apoptosis and mitochondrial fission remains a challenging question. A large body of evidence demonstrates that mitochondrial fission is involved in the initiation of apoptosis (Cereghetti et al., 2010; Karbowski, 2010). Mitochondrial structural changes occur in response to a variety of apoptotic stimuli. The tubular mitochondria change into small and round ones during apoptosis
(Frank et al., 2001). Concomitantly, Drp1 and hFis1 levels are elevated in early apoptosis in cultured cardiomyocytes (Parra et al., 2008; Ong et al., 2010) and in heart failure model as well (Chen et al., 2009). Drp1 (Frank et al., 2001; Lee et al., 2004) and hFis1 (Lee et al., 2004; Alirol et al., 2006) participate in the process of cell death. During cardiac myocytes apoptosis, Drp1 translocates to mitochondria from cytosol (Wang et al., 2011a). Hitherto, much of mitochondrial fission in the cascades of apoptosis remains to be explored (Fig. 2).

\section{REACTIVE OXYGEN SPECIES (ROS) PLAY AN IMPORTANT ROLE IN REGULATING THE MITOCHONDRIAL NETWORK}

\section{ROS generation in the cardiomyocytes}

ROS include superoxide, hydroxyl radicals and hydrogen peroxide, which are all by-products of normal mitochondrial metabolism and bioenergetic activities. Mitochondrial electron transport chain (ETC) is the major source of ROS production. ETC is composed of inner mitochondria membrane complex I, II, III and IV. It can be injured by cardiac ischemia (Casademont and Miró, 2002; Chen et al., 2008). The impairment of ETC increases the production of ROS, and the excessive superoxide attacks the ironsulfur center of the aconitase, thereby further affecting tricarboxylic acid cycle and the mtDNA (Hausladen and Fridovich, 1994). Cardiomyocyte ETC dysfunction leads to excessive generation of ROS that causes heart malformation and lethality (Ingraham et al., 2009). ROS play an important role in the pathogenesis
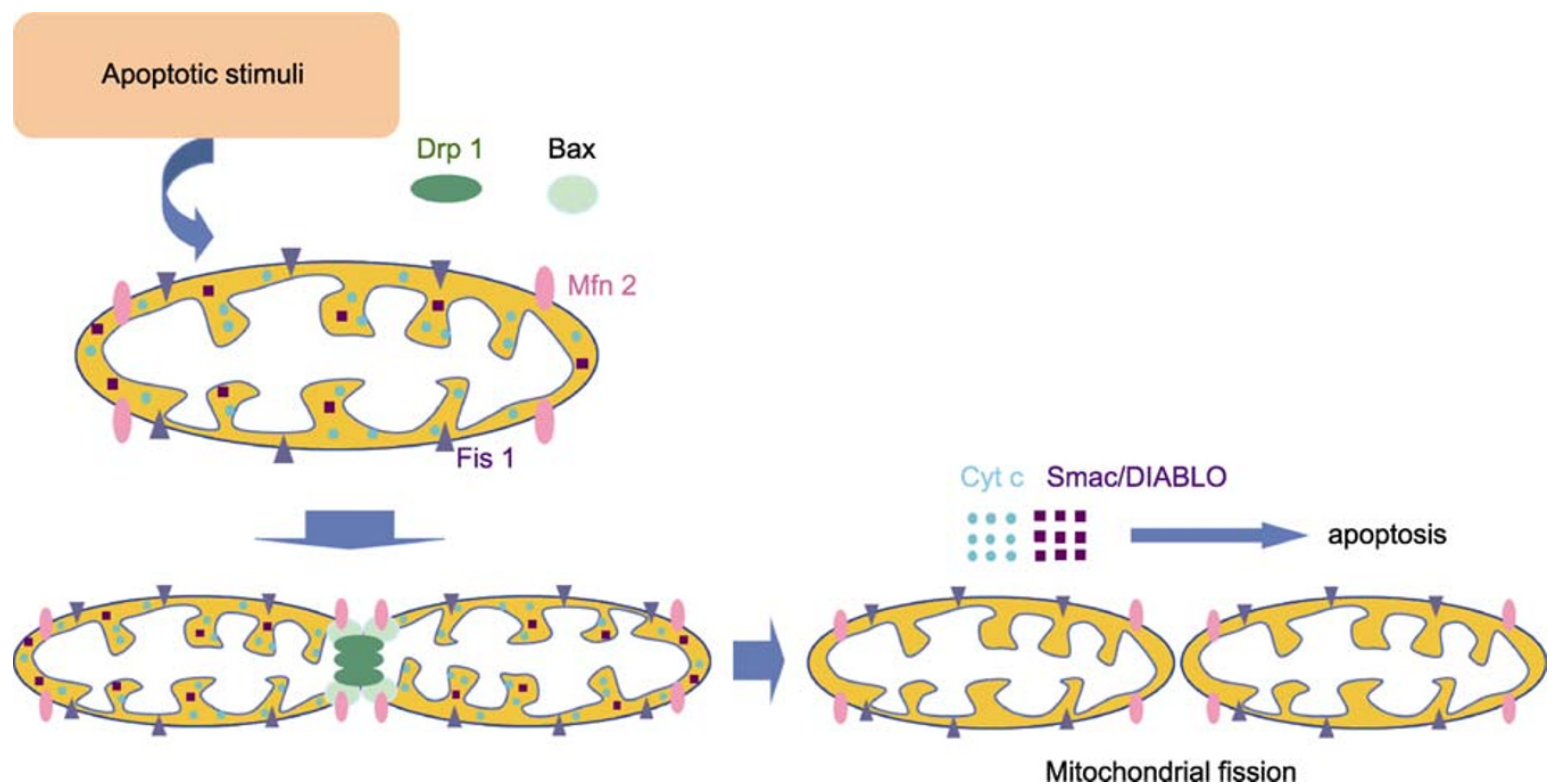

Figure 2. A model of the connection between mitochondrial fission and apoptosis. During apoptosis, Bax and Drp1 translocate to the mitochondria from cytosol and colocalize at the mitochondrial scission sites. They induce mitochondrial fission and the release of cytochrome $c$ as well as Smac/DIABLO. 
of a variety of cardiovascular diseases such as hypertension, cardiomyopathy, cardiac hypertrophy, heart failure and ischemia-reperfusion injury (Ytrehus et al., 1987; Dhalla et al., 2000). Thus, ROS and their origin ETC are vital cellular components in the maintenance of cardiac function.

\section{Central role of ROS in cardiomyocyte apoptosis}

It has long been recognized that ischemia/reperfusion involves not only mitochondria dependent apoptosis, but also necrosis, and ROS functions in both pathways.

ROS are able to induce cardiomyocyte apoptosis in vitro (Aikawa et al., 1997; von Harsdorf et al., 1999). Hydrogen peroxide can induce the translocation of Bax and Bad from cytosol to mitochondria (von Harsdorf et al., 1999). ROS induce the production of TNF-a, which is elevated in many cardiac diseases and cardiomyocyte apoptosis (Suematsu et al., 2003), and ROS may be the direct cause of Cytochrome c release (Atlante et al., 2000). The two recently found ROS generators $p 66^{\text {Shc }}$ and MAO-A are important regulators in ischemia induced cardiomyocyte apoptosis. p66Shc transgenic mice shows greater sensitivity to Angll, in that cardiomyocytes died of apoptosis after treated with Angll (Graiani et al., 2005). p66 ${ }^{\text {Shc }}$ knockout mice have normal blood pressure, heart rate and left ventricular wall thickness, nevertheless, have increased number of cardiomyocytes, and are resistant to the Angll treatment (Graiani et al., 2005). MAO-A inhibitors can reduce the oxidative stress and mitochondrial dependent cell death during ischemia, and finally prevent cardiac damage (Bianchi et al., 2005).

It is now recognizing that apoptosis and necrosis both exist in the ischemia/reperfusion, and contribute differentially to cardiomyocyte injury. Necrosis, rather than apoptosis is the major cause of cell death during ischemia (McCully et al., 2004). Cardiomyocyte apoptosis is evident only at the long term of ischemia (more than 25 minutes), mainly exists adjacent to the ischemia necrotic zone, and inhibition of apoptosis fails to rescue the function of the ischemic cardiomyocyte (Zhao et al., 2000). Apoptosis is largely considered to be triggered during reperfusion (Zhao et al., 2000). ROS and calcium are common factors in necrotic cell death (Vanlangenakker et al., 2008). Mitochondrial calcium overload is the consequence of an elevation of cytosolic calcium during necrosis, resulting in mitochondrial permeability pore (mPTP) opening. And in cardiomyocyte, it is ROS, rather than calcium overload, induce $\mathrm{MPTP}$ opening and result in calcium dysregulation and necrosis (Kim et al., 2006).

\section{ROS affect mitochondrial network}

Mitochondrial morphology is determined by the rate of mitochondrial fission and fusion, and is associated with the generation of ROS (Rodrigues et al., 2011). ROS can induce the opening of mPTP (Zorov et al., 2000), disrupt mi- tochondrial depolarization (Skulachev et al., 2004), and finally result in mitochondrial fragmentation (Zorov et al., 2000; Zhang et al., 2009). Mitochondrial damages, such as photo damage or the mitochondria-targeted oxidants, induce a boost of ROS, cause the loss of membrane potential and interruption of mitochondrial fission (Mai et al., 2010; Schmidt et al., 2010; Mai et al., 2012). Ischemia/reperfusion-induced endothelial cell mitochondrial fission is related to mitochondrial ROS generation (Giedt et al., 2012; Johnson et al., 2012).

Many mitochondrial fission/fusion factors play a role in ROS production. Drp1 promote the production of ROS (Ferrari et al., 2011). Knockdown of Drp1 delays the ROS production (Kobashigawa et al., 2011). Drp1 and ROS mediate the methamphetamine-induced mitochondrial fission (Tian et al., 2009). Mfn-1 remodels the outer mitochondrial membrane in mitochondrial fusion. In the cardiomyocyte-specific Mfn-1 knockout mice, mitochondrial fusion is reduced and cardiomyocytes exhibit increasing resistance against the imminent ROS overload and ROS-induced mitochondrial dysfunction (Papanicolaou et al., 2012). Pink1 is a mitochondria-targeted Ser/Thr kinase. It genetically interacts with the mitochondrial fission/fusion machinery (Yang et al., 2008) and participates in a FOXO3a-mediated ROS defense pathway (Mei et al., 2009). Pink1 knockout results in respiratory dysfunction, ROS elevation (Wood-Kaczmar et al., 2008), and mitochondrial fission (Chu, 2010) (Fig. 3).

\section{PERSPECTIVE}

Recent studies have revealed that mitochondria are highly dynamic organelles that continuously undergo fission and fusion to form a tubular network within the cell. Mitochondrial network constantly changes in respond to different physical and biological environment, and it plays a crucial role in the maintenance of cell integrity. Over the past years, several lines of evidence have shown that proteins or miRNAs can modulate mitochondrial dynamics. However, how individual mitochondria communicate with neighbor ones or the ones over distance to coordinate as a network in response to stress remains to be elucidated. It is likely that the study on mitochondrial dynamics will provide groundbreaking discoveries in the field of cell biology and preclinical medicine, as it holds a promising therapeutic means in treating heart disease.

\section{ABBREVIATIONS}

ARC, apoptosis repressor with caspase recruitment domain; BAR, bifunctional apoptosis regulator; Drp1, Dynamin-related protein 1; ETC, electron transport chain; IFM, interfibrillar mitochondria; Mfn, mitofusin; Pink1, putative kinase 1; ROS, reactive oxygen species; OPA1, optic atrophy-1; SSM, subsarcolemmal mitochondria; ULK1, UNC51-like kinase1 


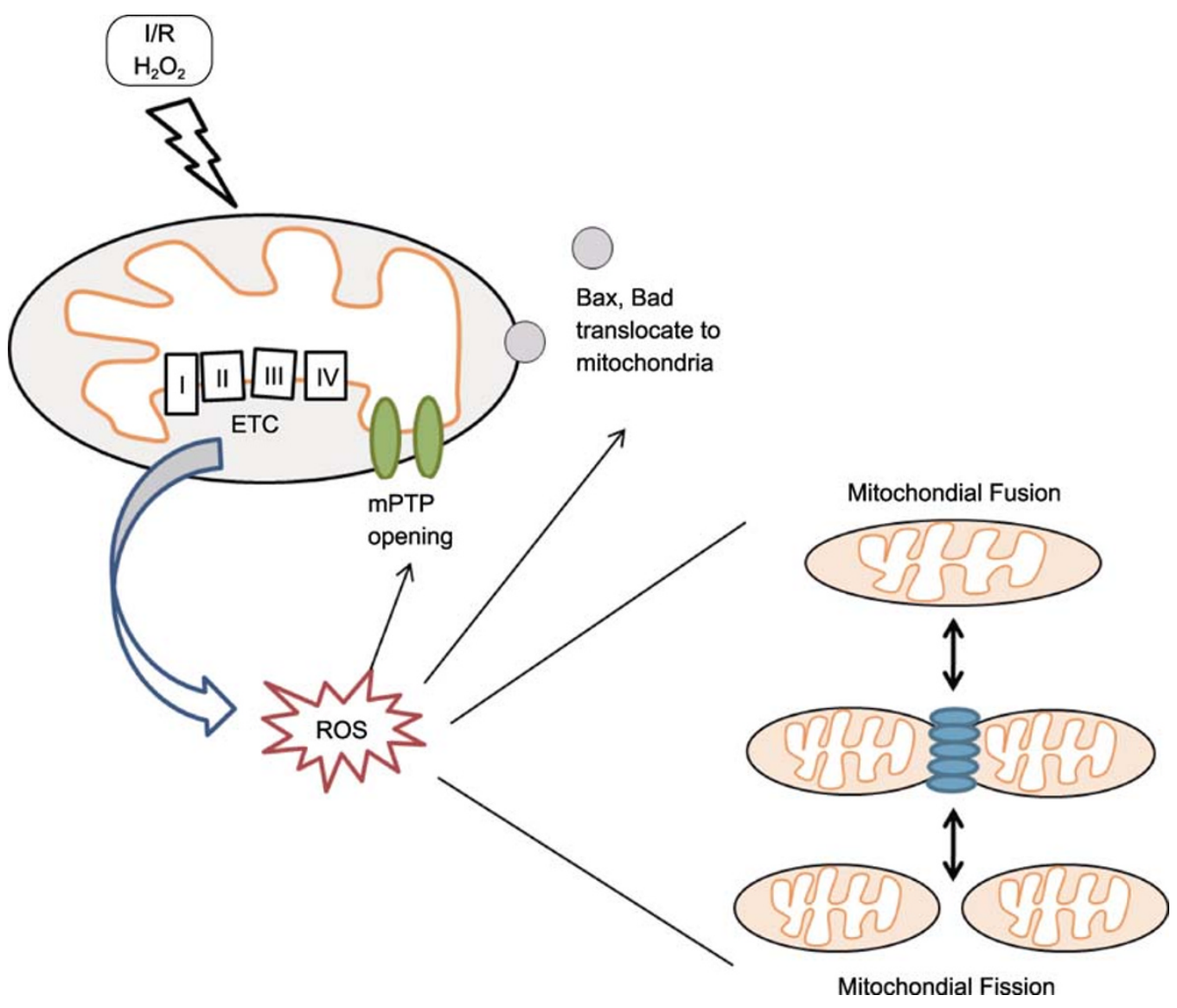

Figure 3. The schematic map of ROS in association with mitochondrial fission and fusion. ROS elevation leads to opening of mPTP, disrupts mitochondrial depolarization, and finally results in mitochondrial fragmentation.

\section{REFERENCES}

Adams, J.M., and Cory, S. (2001). Life-or-death decisions by the Bcl-2 protein family. Trends Biochem Sci 26, 61-66.

Aharinejad, S., Andrukhova, O., Lucas, T., Zuckermann, A., Wieselthaler, G., Wolner, E., and Grimm, M. (2008). Programmed cell death in idiopathic dilated cardiomyopathy is mediated by suppression of the apoptosis inhibitor Apollon. Ann Thorac Surg 86, 109-114.

Aikawa, R., Komuro, I., Yamazaki, T., Zou, Y., Kudoh, S., Tanaka, M., Shiojima, I., Hiroi, Y., and Yazaki, Y. (1997). Oxidative stress activates extracellular signal-regulated kinases through $\mathrm{Src}$ and Ras in cultured cardiac myocytes of neonatal rats. J Clin Invest 100, 1813-1821.

Alirol, E., James, D., Huber, D., Marchetto, A., Vergani, L., Martinou, J.C., and Scorrano, L. (2006). The mitochondrial fission protein $\mathrm{hFis} 1$ requires the endoplasmic reticulum gateway to induce apoptosis. Mol Biol Cell 17, 4593-4605.

Atlante, A., Calissano, P., Bobba, A., Azzariti, A., Marra, E., and Passarella, S. (2000). Cytochrome $c$ is released from mitochondria in a reactive oxygen species (ROS)-dependent fashion and can operate as a ROS scavenger and as a respiratory substrate in cerebellar neurons undergoing excitotoxic death.
Journal of Biological Chemistry 275, 37159-37166.

Baines, C.P. (2010). The cardiac mitochondrion: nexus of stress. Annu Rev Physiol 72, 61-80.

Bianchi, P., Kunduzova, O., Masini, E., Cambon, C., Bani, D., Raimondi, L., Seguelas, M.H., Nistri, S., Colucci, W., and Leducq, N. (2005). Oxidative stress by monoamine oxidase mediates receptor-independent cardiomyocyte apoptosis by serotonin and postischemic myocardial injury. Circulation 112, 3297-3305.

Brocheriou, V., Hagège, A.A., Oubenaïssa, A., Lambert, M., Mallet, V.O., Duriez, M., Wassef, M., Kahn, A., Menasché, P., and Gilgenkrantz, H. (2000). Cardiac functional improvement by a human $\mathrm{Bcl}-2$ transgene in a mouse model of ischemia/reperfusion injury. J Gene Med 2, 326-333.

Bryant, D., Becker, L., Richardson, J., Shelton, J., Franco, F., Peshock, R., Thompson, M., and Giroir, B. (1998). Cardiac failure in transgenic mice with myocardial expression of tumor necrosis factor-a. Circulation 97, 1375-1381.

Casademont, J., and Miró, Ò. (2002). Electron transport chain defects in heart failure. Heart Fail Rev 7, 131-139.

Cereghetti, G., Costa, V., and Scorrano, L. (2010). Inhibition of Drp1-dependent mitochondrial fragmentation and apoptosis by a polypeptide antagonist of calcineurin. Cell Death Differ 17, 1785-1794. 
Chen, H., and Chan D.C. (2004). Mitochondrial dynamics in mammals. Curr Top Dev Biol 59, 119-144.

Chen, L., Gong, Q., Stice, J.P., and Knowlton, A.A. (2009). Mitochondrial OPA1, apoptosis, and heart failure. Cardiovasc Res 84, 91-99.

Chen, Q., Moghaddas, S., Hoppel, C.L., and Lesnefsky, E.J. (2008). Ischemic defects in the electron transport chain increase the production of reactive oxygen species from isolated rat heart mitochondria. Am J Physiol Cell Physiol 294, C460-C466.

Chen, Z., Chua, C.C., Ho, Y.S., Hamdy, R.C., and Chua, B.H.L. (2001). Overexpression of Bcl-2 attenuates apoptosis and protects against myocardial I/R injury in transgenic mice. Am J Physiol Cell Physiol 280, H2313-H2320.

Chu, C.T. (2010). Tickled PINK1: mitochondrial homeostasis and autophagy in recessive Parkinsonism. Biochim Biophys Acta 1802, 20-28.

Chua, C.C., Gao, J., Ho, Y.S., Xu, X., Kuo, I., Chua, K.Y., Wang, H., Hamdy, R.C., Reed, J.C., and Chua, B.H.L. (2009). Over-expression of a modified bifunctional apoptosis regulator protects against cardiac injury and doxorubicin-induced cardiotoxicity in transgenic mice. Cardiovasc Res 81, 20-27.

Cipolat, S., Martins de Brito, O., Dal Zilio, B. \& Scorrano, L. (2004). OPA1 requires mitofusin 1 to promote mitochondrial fusion. Proc Natl Acad Sci U S A 101, 15927-15932.

Cribbs, J.T., and Strack, S. (2007). Reversible phosphorylation of Drp1 by cyclic AMP-dependent protein kinase and calcineurin regulates mitochondrial fission and cell death. EMBO Rep 8, 939-944.

Detmer S.A., and Chan, D. (2007). Functions and dysfunctions of mitochondrial dynamics. Mol Cell Biol 8, 870-879.

Dhalla, N.S., Temsah, R.M., and Netticadan, T. (2000). Role of oxidative stress in cardiovascular diseases. J Hypertens 18, 655-673.

Diwan, A., Wansapura, J., Syed, F.M., Matkovich, S.J., Lorenz, J.N., and Dorn, G.W. (2008). Nix-mediated apoptosis links myocardial fibrosis, cardiac remodeling, and hypertrophy decompensation. Circulation 117, 396-404.

Donath, S., Li, P., Willenbockel, C., Al-Saadi, N., Gross, V., Willnow, T., Bader, M., Martin, U., Bauersachs, J., and Wollert, K.C. (2006). Apoptosis repressor with caspase recruitment domain is required for cardioprotection in response to biomechanical and ischemic stress. Circulation 113, 1203-1212.

Eura, Y., Ishihara, N., Yokota, S., and Mihara, K. (2003). Two mitofusin proteins, mammalian homologues of $\mathrm{FZO}$, with distinct functions are both required for mitochondrial fusion. $\mathrm{J}$ Biochem 134, 333-344.

Ferrari, L.F., Chum, A., Bogen, O., Reichling, D.B., and Levine, J.D. (2011). Role of Drp1, a key mitochondrial fission protein, in neuropathic pain. J Neurosci 31, 11404-11410.

Finck, B.N., and Kelly, D.P. (2007). Peroxisome proliferator-activated receptor gamma coactivator-1 (PGC-1) regulatory cascade in cardiac physiology and disease. Circulation 115, 2540-2548.

Frank, S., Gaume, B., Bergmann-Leitner, E.S., Leitner, W.W., Robert, E.G., Catez, F., Smith, C.L., and Youle, R.J. (2001). The role of dynamin-related protein 1 , a mediator of mitochondrial fission, in apoptosis. Dev Cell 1, 515-525.

Gürtl, B., Kratky, D., Guelly, C., Zhang, L., Gorkiewicz, G., Das, S.K.,
Tamilarasan, K.P., and Hoefler, G. (2009). Apoptosis and fibrosis are early features of heart failure in an animal model of metabolic cardiomyopathy. Int J Exp Pathol 90, 338-346.

Gao, H.K., Yin, Z., Zhou, N., Feng, X.Y., Gao, F., and Wang, H.C. (2008). Glycogen synthase kinase 3 inhibition protects the heart from acute ischemia-reperfusion injury via inhibition of inflammation and apoptosis. J Cardiovasc Pharmacol 52, 286.

Geisler, S., Holmström, K.M., Skujat, D., Fiesel, F.C., Rothfuss, O.C., Kahle, P.J., and Springer, W. (2010). PINK1/Parkin-mediated mitophagy is dependent on VDAC1 and p62/SQSTM1. Nat Cell Biol 12, 119-131.

Giedt, R.J., Yang, C., Zweier, J.L., Matzavinos, A., and Alevriadou, B.R. (2012). Mitochondrial fission in endothelial cells after simulated ischemia/reperfusion: role of nitric oxide and reactive oxygen species. Free Radic Biol Med 52, 348-356.

Gomes, L.C., and Scorrano, L. (2008). High levels of Fis1, a pro-fission mitochondrial protein, trigger autophagy. Biochim Biophys Acta 1777, 860-866.

Graiani, G., Lagrasta, C., Migliaccio, E., Spillmann, F., Meloni, M., Madeddu, P., Quaini, F., Padura, I.M., Lanfrancone, L., and Pelicci, P.G. (2005). Genetic deletion of the p66Shc adaptor protein protects from angiotensin II-induced myocardial damage. Hypertension 46, 433-440.

Griparic L., van der Wel N.N., Orozco I.J., Peters P.J., and van der Bliek A.M. (2004). Loss of the intermembrane space protein Mgm1/OPA1 induces swelling and localized constrictions along the lengths of mitochondria. J Biol Chem 279, 18792-18798.

Gustafsson, Å.B., and Gottlieb, R.A. (2008). Heart mitochondria: gates of life and death. Cardiovasc Res 77, 334.

Hailey, D.W., Rambold, A.S., Satpute-Krishnan, P., Mitra, K., Sougrat, R., Kim, P.K., and Lippincott-Schwartz, J. (2010). Mitochondria supply membranes for autophagosome biogenesis during starvation. Cell 141, 656-667.

Hausladen, A., and Fridovich, I. (1994). Superoxide and peroxynitrite inactivate aconitases, but nitric oxide does not. J Biol Chem 269, 29405-29408.

Hom, J., and Sheu, S.S. (2009). Morphological dynamics of mitochondria--a special emphasis on cardiac muscle cells. J Mol Cell Cardiol 46, 811-820.

Hom, J., Yu, T., Yoon, Y., Porter, G., and Sheu, S.S. (2010). Regulation of mitochondrial fission by intracellular $\mathrm{Ca} 2+$ in rat ventricular myocytes. Biochim Biophys Acta 1797, 913-921.

Hoppela C. L., Tandlerb B., Fujiokac H., and Rivad A. (2009). Dynamic organization of mitochondria in human heart and in myocardial disease. Int J Biochem Cell Biol 41, 1949-1956.

Huss, J.M., and Kelly, D.P. (2005). Mitochondrial energy metabolism in heart failure: a question of balance. J Clin Invest 115, 547-555.

Hyde, B.B., Twig, G., and Shirihai, O.S. (2010). Organellar vs cellular control of mitochondrial dynamics. Semin Cell Dev Biol 21, 575-581.

Imahashi, K., Schneider, M.D., Steenbergen, C., and Murphy, E. (2004). Transgenic expression of Bcl-2 modulates energy metabolism, prevents cytosolic acidification during ischemia, and reduces ischemia/reperfusion injury. Circ Res 95, 734-741.

Ingraham, C.A., Burwell, L.S., Skalska, J., Brookes, P.S., Howell, R.L., Sheu, S.S., and Pinkert, C.A. (2009). NDUFS4: creation of a mouse model mimicking a Complex I disorder. Mitochondrion 9, 
204-210.

Jofuku, A., Ishihara, N., and Mihara, K. (2005). Analysis of functional domains of rat mitochondrial Fis1, the mitochondrial fissionstimulating protein. Biochem Biophys Res Commun 333, 650-659.

Johnson, D., Allman, E., and Nehrke, K. (2012). Regulation of acid-base transporters by reactive oxygen species following mitochondrial fragmentation. Am J Physiol Cell Physiol, 302 C1045-C1054.

Karbowski, M. (2010). Mitochondria on guard: role of mitochondrial fusion and fission in the regulation of apoptosis BCL-2 protein family. In, C. Hetz, ed. (Springer New York), pp. 131-142.

Kim, I., Rodriguez-Enriquez, S. and Lemasters, J.J. (2007). Selective degradation of mitochondria by mitophagy. Arch Biochem Biophys 245-253.

Kim, J.S., Jin, Y., and Lemasters, J.J. (2006). Reactive oxygen species, but not $\mathrm{Ca} 2+$ overloading, trigger $\mathrm{pH}$-and mitochondrial permeability transition-dependent death of adult rat myocytes after ischemia-reperfusion. Am J Physiol Heart Circ Physiol 290, H2024-H2034.

Kobashigawa, S., Suzuki, K., and Yamashita, S. (2011). lonizing radiation accelerates Drp1-dependent mitochondrial fission, which involves delayed mitochondrial reactive oxygen species production in normal human fibroblast-like cells. Biochem Biophys Res Commun 414, 795-800.

Kroemer, G., Galluzzi, L., and Brenner, C. (2007). Mitochondrial membrane permeabilization in cell death. Physiol Rev 87, 99-163.

Kundu, M., Lindsten, T., Yang, C.Y., Wu, J., Zhao, F., Zhang, J., Selak, M.A., Ney, P.A., and Thompson, C.B. (2008). Ulk1 plays a critical role in the autophagic clearance of mitochondria and ribosomes during reticulocyte maturation. Blood 112, 1493-1502.

Lee, Y., Jeong, S.Y., Karbowski, M., Smith, C.L., and Youle, R.J. (2004). Roles of the mammalian mitochondrial fission and fusion mediators Fis1, Drp1, and Opa1 in apoptosis. Mol Biol Cell 15, 5001-5011.

Lee, Y., Lee, H.Y., Hanna, R.A., and Gustafsson, Å.B. (2011). Mitochondrial autophagy by Bnip3 involves Drp1-mediated mitochondrial fission and recruitment of Parkin in cardiac myocytes. Am J Physiol Heart Circ Physiol 301, H1924-H1931.

Legros, F., Lombès, A., Frachon, P., and Rojo, M. (2002). Mitochondrial fusion in human cells is efficient, requires the inner membrane potential, and is mediated by mitofusins,. Mol Biol Cell 13, 4343-4354.

Lehman, J.J., Barger, P.M., Kovacs, A., Saffitz, J.E., Medeiros, D.M., and Kelly, D.P. (2000). Peroxisome proliferator-activated receptor gamma coactivator-1 promotes cardiac mitochondrial biogenesis. $\mathrm{J}$ Clin Invest 106, 847-856.

Li, J., Donath, S., Li, Y., Qin, D., Prabhakar, B.S., and Li, P. (2010). miR-30 regulates mitochondrial fission through targeting p53 and the dynamin-related protein-1 pathway. PLoS Genet 6, e1000795.

Li, P.F., Li, J., Muller, E.C., Otto, A., Dietz, R., and von Harsdorf, R. (2002). Phosphorylation by protein kinase CK2: a signaling switch for the caspase-inhibiting protein ARC. Mol Cell 10, 247-258.

Lin, K.M., Lin, B., Lian, I.Y., Mestril, R., Scheffler, I.E., and Dillmann, W.H. (2001). Combined and individual mitochondrial HSP60 and HSP10 expression in cardiac myocytes protects mitochondrial function and prevents apoptotic cell deaths induced by simulated ischemia-reoxygenation. Circulation 103, 1787-1792.

Mai, S., Klinkenberg, M., Auburger, G., Bereiter-Hahn, J., and Jendrach, M. (2010). Decreased expression of Drp1 and Fis1 mediates mitochondrial elongation in senescent cells and enhances resistance to oxidative stress through PINK1. J Cell Sci 123, 917-926.

Mai, S., Muster, B., Bereiter-Hahn, J., and Jendrach, M. (2012). Autophagy proteins LC3B, ATG5 and ATG12 participate in quality control after mitochondrial damage and influence lifespan. Autophagy 8, 47-62.

Matsui, Y., Takagi, H., Qu, X., Abdellatif, M., Sakoda, H., Asano, T., Levine, B., and Sadoshima, J. (2007). Distinct roles of autophagy in the heart during ischemia and reperfusion. Circ Res 100, 914-922.

Mayor, F., and Cuezva, J.M. (1985). Hormonal and metabolic changes in the perinatal period. Biol Neonate 48, 185-196.

McCully, J.D., Wakiyama, H., Hsieh, Y.J., Jones, M., and Levitsky, S. (2004). Differential contribution of necrosis and apoptosis in myocardial ischemia-reperfusion injury. Am J Physiol Heart Circ Physiol 286, H1923-H1935.

Mei, Y., Zhang, Y., Yamamoto, K., Xie, W., Mak, T.W., and You, H. (2009). FOXO3a-dependent regulation of Pink1 (Park6) mediates survival signaling in response to cytokine deprivation. Proc Natl Acad Sci U S A 106, 5153-5158.

Meeusen, S., McCaffery, J.M., and Nunnari, J. (2004). Mitochondrial fusion intermediates revealed in vitro. Science 305, 1747-1752.

Misao, J., Hayakawa, Y., Ohno, M., Kato, S., Fujiwara, T., and Fujiwara, H. (1996). Expression of bcl-2 protein, an inhibitor of apoptosis, and Bax, an accelerator of apoptosis, in ventricular myocytes of human hearts with myocardial infarction. Circulation 94, 1506-1512.

Mizushima, N., Levine, B., Cuervo, A.M., and Klionsky D.J. (2008). Autophagy fights disease through cellular self-digestion. Nature 451, 1069-1075.

Mortensen, M., Ferguson, D.J., Edelmann, M., Kessler, B., Morten, K.J., Komatsu, M., and Simon, A.K. (2010). Loss of autophagy in erythroid cells leads to defective removal of mitochondria and severe anemia in vivo. Proc Natl Acad Sci U S A 107, 832-837.

Mouli P.K., Twig, G., and Shirihai, O.S. (2009). Frequency and selectivity of mitochondrial fusion are key to its quality maintenance function. Biophys J 96, 3509-3518.

Nakai, A., Yamaguchi, O., Takeda, T., Higuchi, Y., Hikoso, S., Taniike, M., Omiya, S., Mizote, I., Matsumura, Y., Asahi M., et al. (2007). The role of autophagy in cardiomyocytes in the basal state and in response to hemodynamic stress. Nat Med 13, 619-624.

Olivetti, G., Quaini, F., Sala, R., Lagrasta, C., Corradi, D., Bonacina, E., Gambert, S.R., Cigola, E., and Anversa, P. (1996). Acute myocardial infarction in humans is associated with activation of programmed myocyte cell death in the surviving portion of the heart. J Mol Cell Cardiol 28, 2005-2016.

Ong, S.B., Subrayan, S., Lim, S.Y., Yellon, D.M., Davidson, S.M., and Hausenloy, D.J. (2010). Inhibiting mitochondrial fission protects the heart against ischemia/reperfusion injury. Circulation 121, 2012-2022.

Papanicolaou, K.N., Ngoh, G.A., Dabkowski, E.R., O'Connell, K.A., Ribeiro, R.F., Jr., Stanley, W.C., and Walsh, K. (2012). 
Cardiomyocyte deletion of mitofusin-1 leads to mitochondrial fragmentation and improves tolerance to ROS-induced mitochondrial dysfunction and cell death. Am J Physiol Heart Circ Physiol 302, H167-H179.

Parra, V., Eisner, V., Chiong, M., Criollo, A., Moraga, F., Garcia, A., Härtel, S., Jaimovich, E., Zorzano, A., and Hidalgo, C. (2008). Changes in mitochondrial dynamics during ceramide-induced cardiomyocyte early apoptosis. Cardiovas Rev 77, 387-397.

Rodrigues, R.M., Macko, P., Palosaari, T., and Whelan, M.P. (2011). Autofluorescence microscopy: a non-destructive tool to monitor mitochondrial toxicity. Toxicol Lett 206, 281-288.

Schaper, J., Froede, R., Hein, S., Buck, A., Hashizume, H., Speiser, B., Friedl, A., and Bleese, N. (1991). Impairment of the myocardial ultrastructure and changes of the cytoskeleton in dilated cardiomyopathy. Circulation 83, 504-514.

Schmidt, S.P., Corydon, T.J., Pedersen, C.B., Bross, P., and Gregersen, N. (2010). Misfolding of short-chain acyl-CoA dehydrogenase leads to mitochondrial fission and oxidative stress. Mol Genet Metab 100, 155-162.

Schweers, R.L., Zhang, J., Randall, M.S., Loyd, M.R., Li, W., Dorsey, F.C., Kundu, M., Opferman, J.T., Cleveland, J.L., Miller, J.L., et al. (2007). NIX is required for programmed mitochondrial clearance during reticulocyte maturation. Proc Natl Acad Sci U S A 104, 19500-19505.

Skulachev, V.P., Bakeeva, L.E., Chernyak, B.V., Domnina, L.V., Minin, A.A., Pletjushkina, O.Y., Saprunova, V.B., Skulachev, I.V., Tsyplenkova, V.G., and Vasiliev, J.M. (2004). Thread-grain transition of mitochondrial reticulum as a step of mitoptosis and apoptosis. Mol Cell Biochem 256, 341-358.

Stojanovski D., Koutsopoulos O.S., Okamoto K., and Ryan M.T. (2004). Levels of human Fis1 at the mitochondrial outer membrane regulate mitochondrial morphology. J Cell Sci 117, 1201-1210.

Suematsu, N., Tsutsui, H., Wen, J., Kang, D., Ikeuchi, M., Ide, T., Hayashidani, S., Shiomi, T., Kubota, T., and Hamasaki, N. (2003). Oxidative stress mediates tumor necrosis factor- $\alpha$-induced mitochondrial DNA damage and dysfunction in cardiac myocytes. Circulation 107, 1418-1423.

Tian, C., Murrin, L.C., and Zheng, J.C. (2009). Mitochondrial fragmentation is involved in methamphetamine-induced cell death in rat hippocampal neural progenitor cells. PLoS One 4, e5546.

Tolkovsky, A.M., Xue, L., Fletcher, G.C., and Borutaite, V. (2002). Mitochondrial disappearance from cells: a clue to the role of autophagy in programmed cell death and disease? Biochimie 84, 233-240.

Twig G., Elorza A., Molina A.J., Mohamed H., Wikstrom J.D., Walzer G., Stiles L., Haigh S.E., Katz S., Las G., et al. (2008a). Fission and selective fusion govern mitochondrial segregation and elimination by autophagy. EMBO J 27, 433-446.

Twig G., Hyde B., Shirihai O.S., (2008b). Mitochondrial fusion, fission and autophagy as a quality control axis: the bioenergetic view. Biochim Biophys Acta 1777, 1092-1097.

Vanlangenakker, N., Berghe, T.V., Krysko, D.V., Festjens, N., and Vandenabeele, P. (2008). Molecular mechanisms and pathophysiology of necrotic cell death. Curr Mol Med 8, 207-220.

von Harsdorf, R., Li, P.F., and Dietz, R. (1999). Signaling pathways in reactive oxygen species-induced cardiomyocyte apoptosis. Circulation 99, 2934-2941.

Wang, J.X., Jiao, J.Q., Li, Q., Long, B., Wang, K., Liu, J.P., Li, Y.R., and Li, P.F. (2011a). miR-499 regulates mitochondrial dynamics by targeting calcineurin and dynamin-related protein-1. Nat Med $17,71-78$.

Wang, J.X., Jiao, J.Q., Li, Q., Long, B., Wang, K., Liu, J.P., Li, Y.R., and Li, P.F. (2011b). miR-499 regulates mitochondrial dynamics by targeting calcineurin and dynamin-related protein-1. Nat Med 17, 71-78.

White, K.E., Davies, V.J., Hogan, V.E., Piechota, M.J., Nichols, P.P., Turnbull, D.M., and Votruba, M. (2009). OPA1 deficiency associated with increased autophagy in retinal ganglion cells in a murine model of dominant optic atrophy. Invest Ophthalmol Vis Sci 50, 2567.

Wood-Kaczmar, A., Gandhi, S., Yao, Z., Abramov, A.S.Y., Miljan, E.A., Keen, G., Stanyer, L., Hargreaves, I., Klupsch, K., and Deas, E. (2008). PINK1 is necessary for long term survival and mitochondrial function in human dopaminergic neurons. PLoS One 3, e2455.

Yang, Y., Ouyang, Y., Yang, L., Beal, M.F., McQuibban, A., Vogel, H., and Lu, B. (2008). Pink1 regulates mitochondrial dynamics through interaction with the fission/fusion machinery. Proc Natl Acad Sci U S A 105, 7070-7075.

Yang Z, and Klionsky D.J. (2010). Eaten alive: a history of macroautophagy. Nature Cell Biol 12, 814-822.

Ytrehus, K., Myklebust, R., Olsen, R., and Mjos, O.D. (1987). Ultrastructural changes induced in the isolated rat heart by enzymatically generated oxygen radicals. J Mol Cell Cardiol 19, 379-389.

Yussman, M.G., Toyokawa, T., Odley, A., Lynch, R.A., Wu, G., Colbert, M.C., Aronow, B.J., Lorenz, J.N., and Dorn, G.W. (2002). Mitochondrial death protein Nix is induced in cardiac hypertrophy and triggers apoptotic cardiomyopathy. Nat Med 8, 725-730.

Zhang, H., Kong, X., Kang, J., Su, J., Li, Y., Zhong, J., and Sun, L. (2009). Oxidative stress induces parallel autophagy and mitochondria dysfunction in human glioma U251 cells. Toxicol Sci 110, 376-388.

Zhao, Z.Q., Nakamura, M., Wang, N.P., Wilcox, J.N., Shearer, S., Ronson, R.S., Guyton, R.A., and Vinten-Johansen, J. (2000). Reperfusion induces myocardial apoptotic cell death. Cardiovasc Res 45, 651-660.

Zhu, H., Tannous, P., Johnstone, J.L., Kong, Y., Shelton, J.M., Richardson, J.A., Le, V., Levine, B., Rothermel, B.A., and Hill, J.A. (2007). Cardiac autophagy is a maladaptive response to hemodynamic stress. J Clin Invest 117, 1782-1793.

Zorov, D.B., Filburn, C.R., Klotz, L.O., Zweier, J.L., and Sollott, S.J. (2000). Reactive oxygen species (ROS)-induced ROS release: a new phenomenon accompanying induction of the mitochondrial permeability transition in cardiac myocytes. J Exp Med 192, 1001-1014. 